\title{
Generation of Optical Vortices by Linear Phase Ramps
}

\author{
Sunil Vyas \\ Institute of Multidisciplinary Research for Advanced Materials, Tohoku University, Katahira 2-1-1, Aoba-ku, Sendai 980-8577, Japan \\ Correspondence should be addressed to Sunil Vyas, sunilk_vyas@yahoo.com
}

Received 11 May 2011; Accepted 17 July 2011

Academic Editor: Paramasivam Senthilkumaran

Copyright ( 2012 Sunil Vyas. This is an open access article distributed under the Creative Commons Attribution License, which permits unrestricted use, distribution, and reproduction in any medium, provided the original work is properly cited.

Generation of optical vortices using linear phase ramps is experimentally demonstrated. When two regions of a wavefront have opposite phase gradients then along the line of phase discontinuity vortices can be generated. It is shown that vortices can evolve during propagation even with the unequal magnitude of tilt in the two regions of the wavefront. The number of vortices and their location depend upon the magnitude of tilt. vortex generation is experimentally realized by encoding phase mask on spatial light modulator and their presence is detected interferometrically. Numerical simulation has been performed to calculate the diffracted intensity distribution from the phase mask, and presence of vortices in the diffracted field is detected by computational techniques.

\section{Introduction}

Optical fields possessing phase singularities or wavefront dislocations have received great deal of attention in recent years because of their fascinating properties and potential applications. The study of phenomena associated with phase singularities has generated a new branch of physical optics called singular optics, which reveals their basic properties and possible applications. Phase singularities also known as optical vortices are the points where the real and imaginary part of wave function vanishes. At phase singular points amplitude is zero and the phase is indeterminate. As a result, the wave front acquires the shape of helicoids, causing the precession of the Poynting vector. Nye and Berry showed that vortices in scalar field are strongly connected with the phase discontinuities or singularities [1]. Near the dislocation centers the phase gradients line is found to form closed vortex-like structures. Optical vortices can appear spontaneously or can be created in one of several ways, such as by the manipulation of laser cavity [2], transforming Gaussian beams into helical beams [3], computer generated holograms [4], cylindrical lenses [5], and spiral phase plates [6]. In recent years several important applications of optical vortices have been demonstrated. For example vortices have been used in optical tweezers [7], wave guiding [8], astronomy as coronagraph [9], optical testing [10], and so forth.
In this paper, experimental and simulation studies are performed to show the formation of vortices along the line of phase discontinuity which arises due to the different phase gradients in the wavefront. To explain the concept of vortex evolution we first discussed a simple case in which the two parts of the wavefront are given equal and opposite phase variations. Diffracted field is analyzed for the vortex evolution using experiments and numerical simulation. Various cases of tilt combinations are also discussed in which the different parts of the wavefront have different magnitude of the phase variations. To check the presence of vortices computational and interferometric techniques have been used. In experiments spatial light modulator is used to provide opposite constant phase gradients of small magnitude at two different regions of the wavefront. It is shown that the generation of single vortex and linear array of vortices using this method is possible. The major advantage of this method is that a vortex of unit charge having phase variation of $2 \pi$ can be comfortably realized by the SLM that can retard wavefront between $\pi$ and $2 \pi$.

\section{Study of Phase Gradients}

2.1. Tilted Plane Wave. A unit amplitude plane wave with tilt is given by

$$
\psi=\exp \{j 2 \pi(\alpha x+\beta y+\gamma z)\}
$$




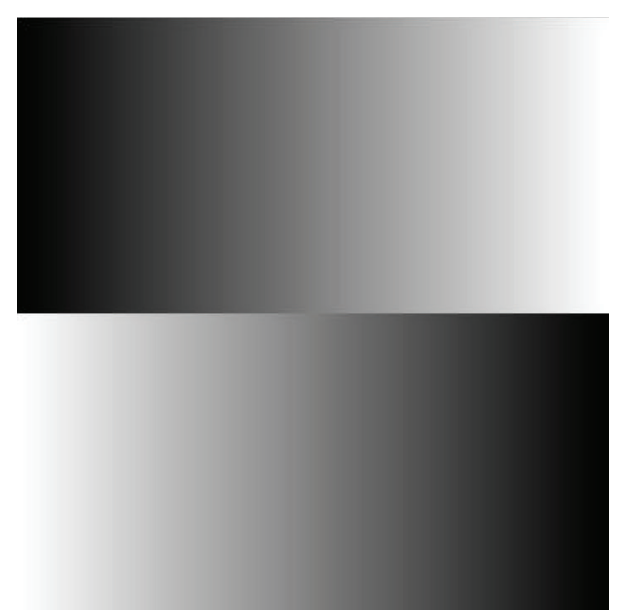

Figure 1: Two linear phase variations in equal and opposite directions in the upper and the lower regions of the wavefront.

where $\alpha, \beta$, and $\gamma$ are spatial frequencies along $x, y$, and $z$ directions, respectively. $\lambda$ is wavelength of light.

The phase gradient for a tilted wavefront is

$$
\nabla \phi=2 \pi(\alpha \hat{x}+\beta \hat{y}+\gamma \hat{z})
$$

Transverse components of phase gradient for tilted plane wave are given by

$$
\nabla_{\perp} \phi=2 \pi(\alpha \hat{x}+\beta \hat{y})
$$

Unlike an optical vortex, here the phase gradient is constant and does not depend on the location.

2.2. Wavefronts with Equal and Opposite Tilts. Consider the case when the two regions in the wavefront have equal and opposite phase variations and the maximum phase variation of the two regions of wavefront is restricted to $\leq 2 \pi$. These two regions are separated by a line of phase discontinuity. The complex amplitude in the two regions is given by

$$
\psi= \begin{cases}\exp (2 \pi \alpha x) & \text { when } y>0 \\ \exp \{(2 \pi \alpha(1-x))\} & \text { when } y<0\end{cases}
$$

where $\alpha$ is a constant which decides the slope of the phase variation across the wavefront. If the wavefront is tilted by angle $\theta$, then $\alpha=\sin \theta / \lambda$ and $\lambda$ is the wavelength of light used. There is a line of phase discontinuity at $y=0$. Figure 1 shows the linear phase distribution of function $\psi_{\mathrm{I}}$. Tilt $\alpha$ gives rate at which the linear phase variation occurs across the beam. Experimentally realizing such small phase variation is easier by using SLM. Detailed study of phase distribution and gradient of phase around the points where the vortices can be evolved is given in [11]. The main objective of this work is to explore the new wavefront tilt configurations for vortex generation.

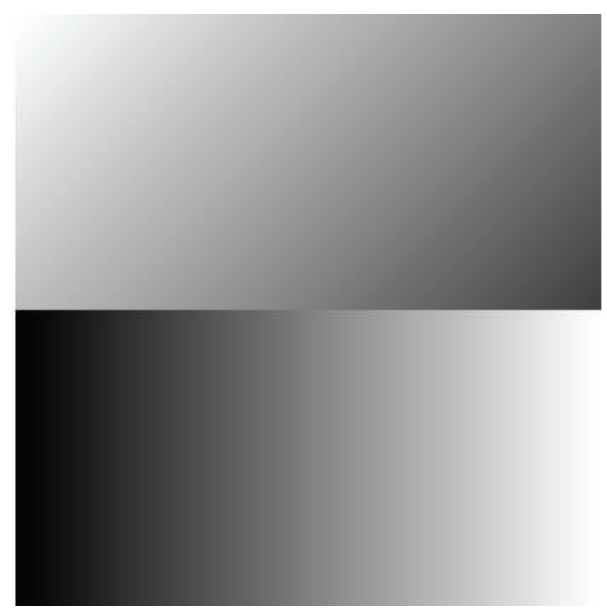

Figure 2: Phase distribution of two wavefronts with unequal and opposite tilts.

2.3. Wavefronts with Unequal and Opposite Tilts. Consider the case when two regions of the wavefront is having unequal and opposite tilts. The complex field can be given as

$$
\psi= \begin{cases}\exp \left(j 2 \pi \alpha_{1} x\right) & \text { when } y \geq 0 \\ \exp \left(j 2 \pi \alpha_{2}(1-x)\right) & \text { when } y \leq 0\end{cases}
$$

The out-of-phase condition for the neighborhood points is well met when opposite phase gradients are present. In this case the $\Delta \phi= \pm \pi$ condition can occur at locations along the line of phase discontinuity $y=0$, whose $x$ coordinates can be given by

$$
\begin{aligned}
& x_{0}=\frac{1+2 \alpha_{2}}{2\left(\alpha_{1}+\alpha_{2}\right)}, \\
& x_{1}=\frac{1-2 \alpha_{2}}{2\left(\alpha_{1}+\alpha_{2}\right)} .
\end{aligned}
$$

One can see the position of points $x_{0}$ and $x_{1}$ has changed when compared with that of previous configuration of wavefront tilts. Figure 2 shows the phase distribution corresponding to tilt equation (5), where $\alpha_{1}$ and $\alpha_{2}$ are constants with different values.

2.4. New Configurations of Phase Structures for Vortex Generation. In the previous cases the phase gradients in the upper and lower parts of the beam point to opposite directions. It is also possible to generate vortices with other configurations of wavefront tilts. To do this let us consider complex field given by

$$
\psi(x, y)= \begin{cases}\exp \left\{j 2 \pi\left(\alpha_{1} x+\beta_{1} y\right)\right\} & \text { for } y>0 \\ \exp \left\{j 2 \pi\left(1-\left(\alpha_{2} x+\beta_{2} y\right)\right)\right\} & \text { for } y<0 .\end{cases}
$$

In the neighborhood of the phase discontinuity line $y=0$, if the $x$-components of the phase gradients are opposite in $y>0$ and $y<0$ regions, it is possible to generate vortices. $\beta_{1}$ and $\beta_{2}$ are constants. 


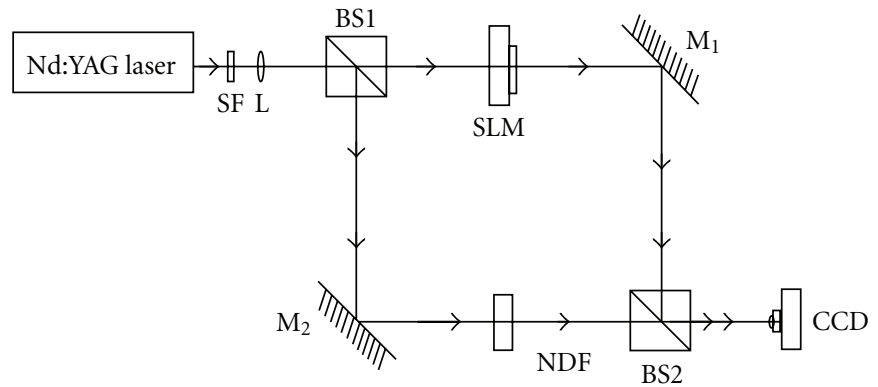

FIGURE 3: Mach-Zehnder interferometer. SF: spatial filter, BS1 and BS2: beam splitters, L: lens, $\mathrm{M}_{1}$ and $\mathrm{M}_{2}$ : mirrors, NDF: neutral density filter, CCD: camera, and SLM: spatial light modulator assembly.

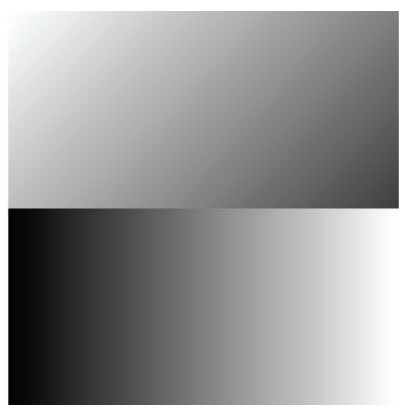

(a)

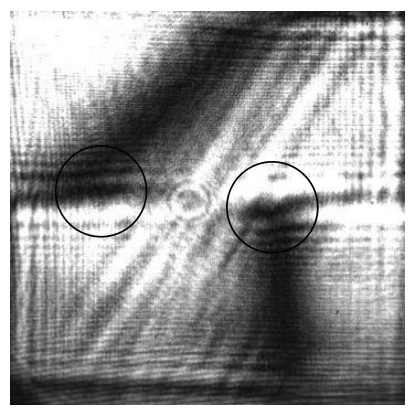

(b)

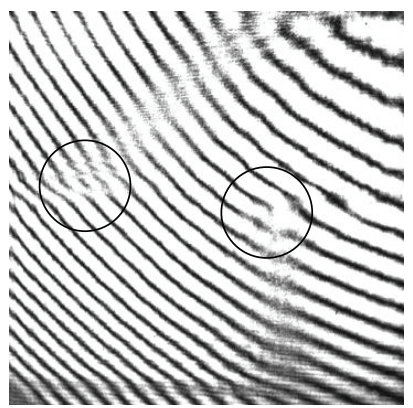

(c)

FIGURE 4: (a) Phase distribution for one oblique and one linear phase ramp. (b) Experimentally observed intensity distribution of propagated field when phase mask corresponding to (a) is displayed onto the SLM. (c) Formation of fork fringes when the propagated field interfers with a plane wave coming from the other arm of the Mach-Zehnder interferometer.

\section{Experimental}

Experiments have been performed to demonstrate the validity of the proposed approach. Optical setup used is shown in Figure 3 in which a Mach-Zehnder interferometer has been used. Output beam of linearly polarized light from a diode pumped solid-state (DPSS) Nd:Yag laser at $532 \mathrm{~nm}$ is spatially filtered, expanded, and collimated by spatial filter and collimating lens, respectively. This light is incident on beam splitter BS1. It splits the incident beam into two parts. One beam is reflected by the mirror M1, which is served as reference beam. The other beam illuminates a programmable spatial light modulator (Holoeye LC-2002 with 832 X624 numbers of pixel and pixel pitch $32 \mu \mathrm{m}$ ) which is operated in phase modulation mode. SLM in phase modulation mode acts like a phase only diffractive optical element. The desired local tilt in the different parts of the wavefront is created by designing the phase mask as grey level images. When the phase masks are encoded onto the SLM and illuminated with the light, it will diffract the light into the different orders. The superposition of the diffracted light from the SLM and the reference plane wave produces interference fringes. To enhance the quality of the interference fringes a neutral density filter is used which makes the amplitude of the reference wave equal to that of the vortex beam.

\section{Experimental Results}

In [11] the phase gradients in the upper and lower parts of the beam point are in opposite directions. In the neighborhood of the phase discontinuity line $y=0$, if the $x$-components of the phase gradients are opposite in $y>0$ and $y<0$ regions, it is possible to generate vortices. Some of the following tilt configurations can generate vortex arrays.

4.1. One Oblique and One Linear Phase Ramp. In this configuration two phase ramps are grouped, one is the slanted and the other is linear as shown in Figure 4(a). The phase distribution in Figure 4 can be obtained by putting $\alpha_{1} \neq 0, \beta_{1} \neq 0$, and $\beta_{2}=0$ in (5). Vortices are positioned at the point where the component of gradient of phase of the slanted wavefront cancels the phase gradient of the linear phase variation. Formation of vortices can be seen as the fork fringes where the exact phase cancellation occurs. It reveals the fact that even if one of the two parts of the wavefront has the different orientation then vortex formation is also possible.

The complex field corresponding to Figure 4(a) is displayed on the SLM, and the intensity distribution obtained is shown in Figure 4(b) which contains two vortices as the interference pattern in Figure 4(c) reveals. 


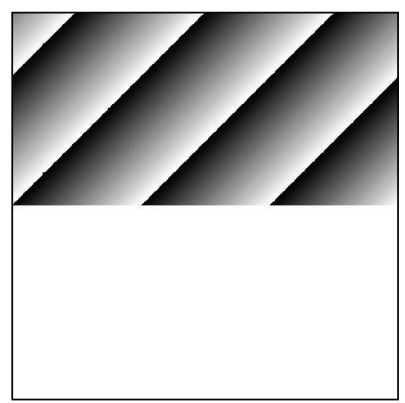

(a)

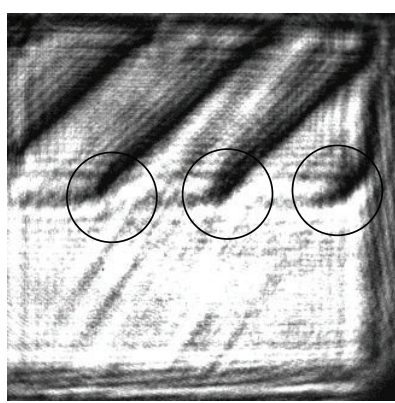

(b)

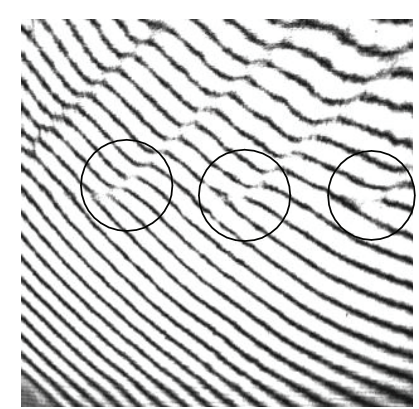

(c)

FIgURe 5: (a) Phase distribution for linear array of inclined phase ramps with constant background. (b) Experimentally observed intensity distribution of propagated field when phase mask corresponding to (a) is displayed onto the SLM. (c) Formation of fork fringes when the propagated field interfers with a plane wave coming from the other arm of the Mach-Zehnder interferometer.

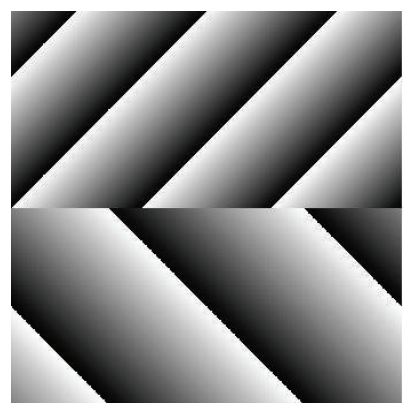

(a)

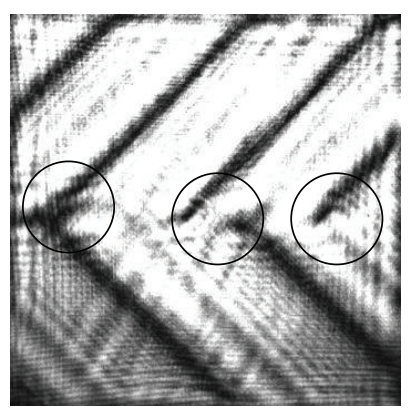

(b)

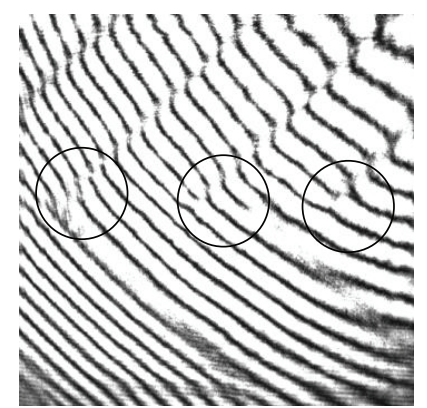

(c)

Figure 6: (a) Phase distribution for oppositely inclined linear phase ramps with different tilts. (b) Experimentally observed intensity distribution of propagated field when phase mask corresponding to (a) is displayed onto the SLM. (c) Formation of fork fringes when the propagated field interfers with a plane wave coming from the other arm of the Mach-Zehnder interferometer.

4.2. Linear Array of Inclined Phase Ramps with Constant Background. In this configuration linear array of vortices is produced by wavefront which has only one part inclined and the other part having constant phase. This case is similar to the case of linear array discussed previously [12]. In this case the number of phase ramps will determine the number of vortices and the slope of the phase ramp will determine the position of vortices.

To obtain phase distribution corresponding to Figure 5(a) $\alpha_{1} \neq 0, \beta_{1} \neq 0$, and $\alpha_{2}=\beta_{2}=0$ are needed. Experimentally observed intensity distribution of propagated field when phase mask corresponding to Figure 5(a) is displayed onto the SLM is shown in Figure 5(b), and the corresponding fork fringes are shown in Figure 5(c), respectively.

\subsection{Oppositely Inclined Linear Phase Ramps with Different} Tilts. Extending the earlier arrangements one more combination of phase ramps is also possible in which two parts of the wavefronts have unequal phase gradients in opposite directions. In this case the formation of three vortices at the point where the components of phase gradients of two parts of the wavefront exactly cancel each others is expected and confirmed. Vortices are detected by the fork fringes in the interference pattern.
In phase distribution for Figure 6(a) $\alpha_{1} \neq 0, \beta_{1} \neq 0$ and $\alpha_{2} \neq 0, \beta_{2} \neq 0$ and further $\alpha_{1} \neq \alpha_{2}$ and $\beta_{1} \neq \beta_{2}$ in (7). Experimentally observed intensity distribution of propagated field when phase mask corresponding to Figure 6(a) is displayed onto the SLM is shown in Figure 6(b), and the corresponding fork fringes are shown in Figure 6(c), respectively. From the above cases it can be seen that at the boundary line separating two regions of wavefront the required opposite gradients can be achieved by component tilts rather than the ones presented earlier in [11].

\section{Simulation Study}

To verify the experimental results numerical studies have been performed. Intensity distribution is computed using Fresnel diffraction formula. The propagation of the complex field from the phase mask is computed by the Fresnel Kirchhoff diffraction integration which is given by [13]

$$
\begin{aligned}
\tilde{U}(x, y)= & \frac{e^{j k z}}{j \lambda z} \iint_{-\infty}^{\infty} \psi \\
& \times \exp \left\{j \frac{k}{2 z}\left[(x-\xi)^{2}+(y-\eta)^{2}\right]\right\} d \xi d \eta,
\end{aligned}
$$




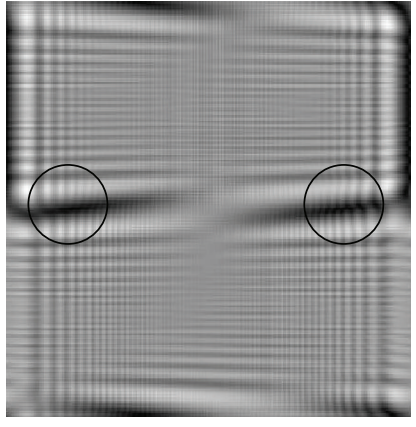

(a)

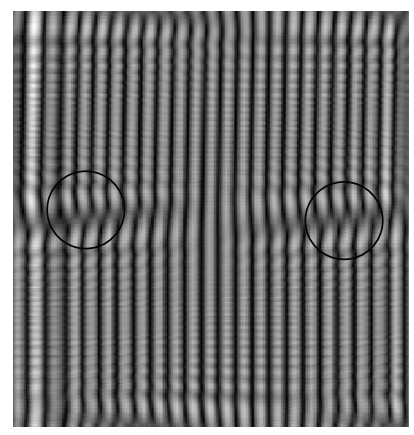

(b)

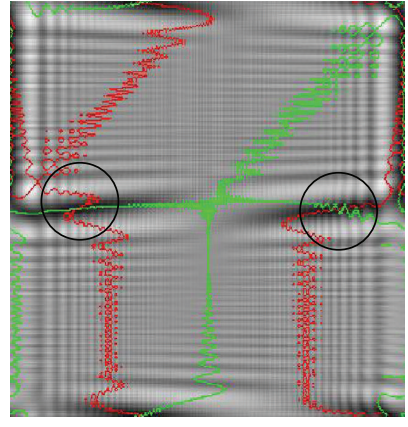

(c)

FIGURE 7: (a) Simulated intensity distribution for one oblique and one linear phase ramp. (b) Simulated interference patterns to check formation of optical vortices. (c) Simulated results for zero crossing of real and imaginary lines one oblique and one linear phase ramp.

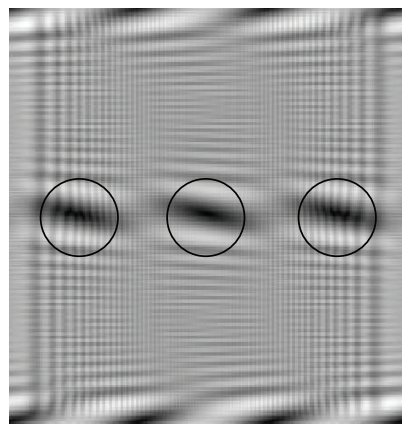

(a)

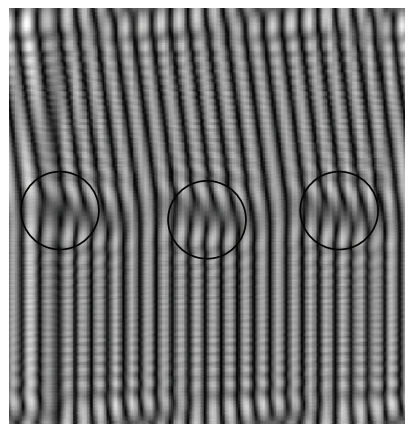

(b)

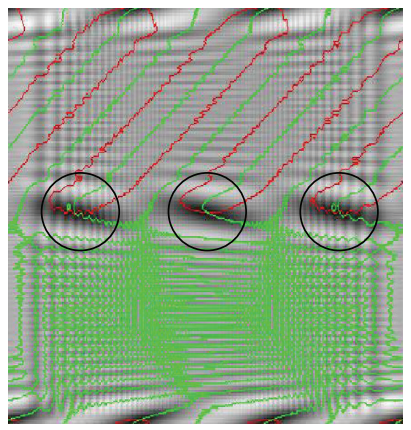

(c)

FIGURE 8: (a) Simulated intensity distribution for linear array of inclined phase ramps with constant background. (b) Simulated interference patterns to check formation of optical vortices. (c) Simulated results for zero crossing of real and imaginary lines for linear array of inclined phase ramps with constant background. Green line represents real part and red line represents imaginary part of the wave function.

where $(x, y)$ and $(\xi, \eta)$ correspond to the object and the diffracted filed planes, respectively. $|k|=2 \pi / \lambda$ is the magnitude of propagation vector, and $z$ is the distance between object and the diffracted plane. Since line phase discontinuity is present in the complex field presented in (8), closed analytical solution of (8) may not be possible for arbitrary values of spatial frequencies in $x$ - and $y$-directions and hence numerical solutions are attempted to view the propagated (diffracted) field. Intensity at the observation plane is

$$
I(x, y)=|\tilde{U}(x, y)|^{2}
$$

Figures 7(a), 8(a), and 9(a) show simulated results for intensity distribution corresponding to the experimentally recorded intensity distribution for unequal tilt configurations. Vortices are located by interfering the vortex beam with a tilted plane wave which forms fork fringes at the singular points which are shown in Figures $7(\mathrm{~b}), 8(\mathrm{~b})$, and $9(\mathrm{~b})$. The exact location of the vortices in the optical field can be obtained by computational methods. One of the important computational methods of vortex detection is the zero crossing method. The zero crossing method is based on the fact that the wave function of the vortex field comprises of real and imaginary parts, both of which vanish simultaneously at the vortex point [14].

Consider a complex field

$$
\tilde{U}(x, y)=a(x, y) e^{i \phi(x, y)} .
$$

The real zero $\operatorname{Re}(0)$ and $\operatorname{Im}(0)$ contours of $\tilde{U}(x, y)$ are given, respectively, as

$$
\begin{aligned}
& a(x, y) \cos (\phi(x, y))=0 \\
& a(x, y) \sin (\phi(x, y))=0 .
\end{aligned}
$$

At the vortex point

$$
a(x, y) \cos (\phi(x, y))=a(x, y) \sin (\phi(x, y))=0
$$

By plotting $\operatorname{Re}(0)$ and $\operatorname{Im}(0)$ lines the presence of vortices can be found by locating the intersection of these lines. Simulated results for zero crossing for Figures 7(a), 8(a), and 9(a) are shown in Figures 7(c), 8(c), and 9(c), respectively. These simulated results match with the experimental results that confirm the presence of vortices at the predicted points. 


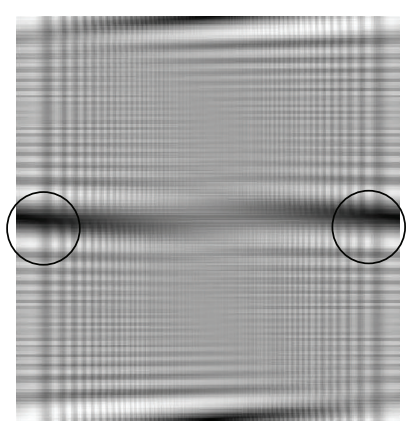

(a)

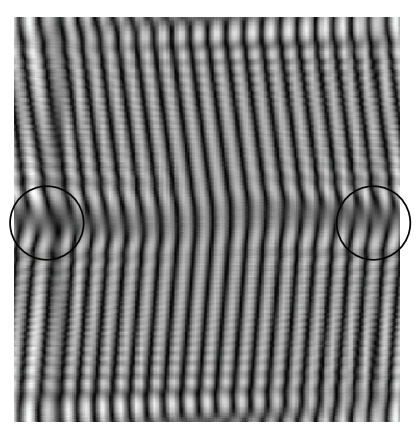

(b)

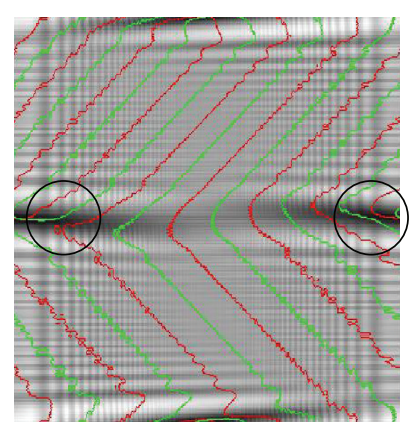

(c)

FIGURE 9: (a) Simulated intensity distribution for linear array for two oppositely inclined phase ramps with different tilt. (b) Simulated interference patterns to check formation of optical vortices. (c) Simulated results for zero crossing of real and imaginary for two oppositely inclined phase ramps with different tilt. In the figure green line represents real part and red line represents imaginary part of the wave function.

\section{Conclusion}

Evolution of vortices in the beam by providing different tilts to the two parts of the wavefront is experimentally demonstrated. For experimental verification tilted wavefronts are generated by encoding the phase mask with linear phase variations on spatial light modulator, and the presence of the vortices in the output field is conformed by formation of characteristic fork and spiral fringes. Different combination of tilts to produce vortices has been described to show the adaptability of the proposed method for vortex array generation. One of the key advantages of this method is the ability to modify optical beam into a symmetric array of vortices dynamically.

\section{Acknowledgment}

The author would like to express his sincere thanks to Professor P. Senthilkumaran for useful discussions and correction of the paper.

\section{References}

[1] J. F. Nye and M. V. Berry, "Dislocations in wave trains," Proceedings of the Royal Society of London Series A, vol. 336, pp. 165-190, 1974.

[2] J. M. Vaughan and D. V. Willetts, "Temporal and interference fringe analysis of TEM $01 *$ laser modes," Journal of the Optical Society of America, vol. 73, no. 8, pp. 1018-1021, 1983.

[3] C. Tamm, "Frequency locking of two transverse optical modes of a laser," Physical Review A, vol. 38, no. 11, pp. 5960-5963, 1988.

[4] N. R. Heckenberg, R. McDuff, C. P. Smith, and A. G. White, "Generation of optical phase singularities by computer generated hologram," Optics Letters, vol. 17, pp. 221-223, 1992.

[5] M. W. Beijersbergen, L. Allen, H. E. L. O. van der Veen, and J. P. Woerdman, "Astigmatic laser mode converters and transfer of orbital angular momentum," Optics Communications, vol. 96, pp. 123-132, 1993.

[6] M. W. Beijersbergen, R. P. C. Coerwinkel, M. Kristensen, and J. P. Woerdman, "Helical-wavefront laser beams produced with a spiral phase plate," Optics Communications, vol. 112, no. 5-6, pp. 321-327, 1994.

[7] K. T. Gahagan and G. A. Swartzlander Jr., "Trapping of lowindex microparticles in an optical vortex," Journal of the Optical Society of America B, vol. 15, no. 2, pp. 524-534, 1998.

[8] G. A. Swartzlander Jr. and C. T. Law, "Optical vortex solitons observed in Kerr nonlinear media," Physical Review Letters, vol. 69, no. 17, pp. 2503-2506, 1992.

[9] J. H. Lee, G. Foo, E. G. Johnson, and G. A. Swartzlander Jr., "Experimental verification of an optical vortex coronagraph," Physical Review Letters, vol. 97, no. 5, article 053901, 2006.

[10] P. Senthilkumaran, "Optical phase singularities in detection of laser beam collimation," Applied Optics, vol. 42, no. 31, pp. 6314-6320, 2003.

[11] S. Vyas and P. Senthilkumaran, "Vortices from wavefront tilts," Optics and Lasers in Engineering, vol. 48, no. 9, pp. 834-840, 2010.

[12] S. Vyas and P. Senthilkumaran, "Two dimensional vortex lattices from pure wavefront tilts," Optics Communications, vol. 283, pp. 2767-2771, 2010.

[13] J. W. Goodman, Introduction to Fourier Optics, The McGrawHill Companies, 2nd edition, 1996.

[14] I. Freund, "Saddles, singularities, and extrema in random phase fields," Physical Review E, vol. 52, no. 3, pp. 2348-2360, 1995. 

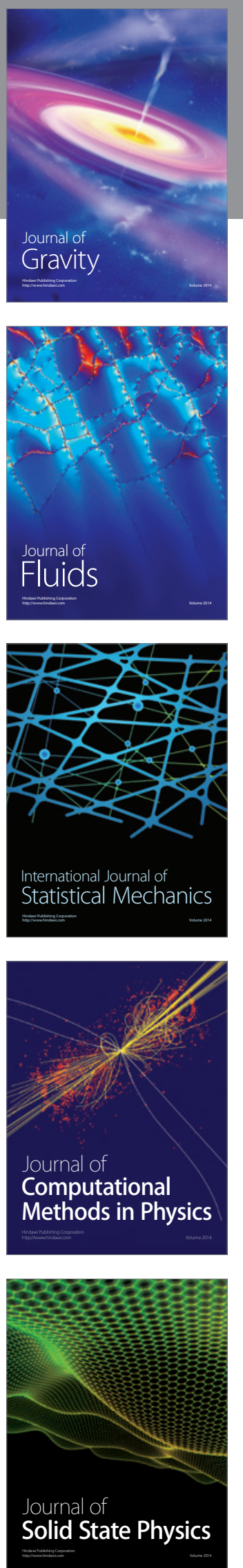

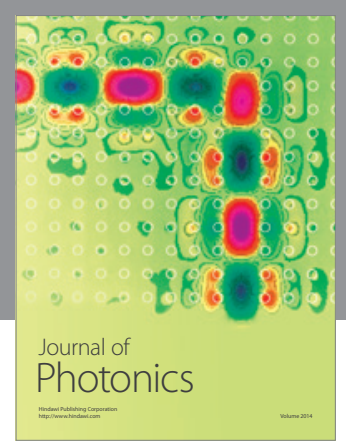

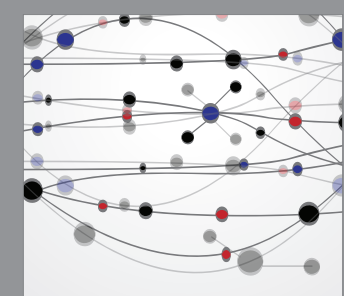

The Scientific World Journal
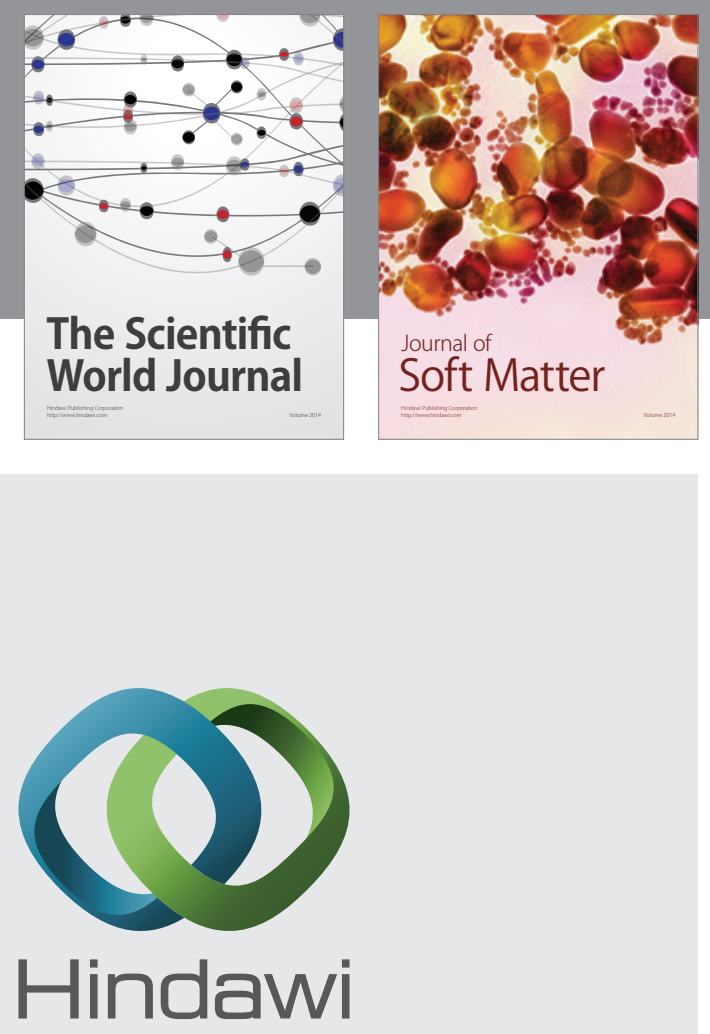

Submit your manuscripts at

http://www.hindawi.com
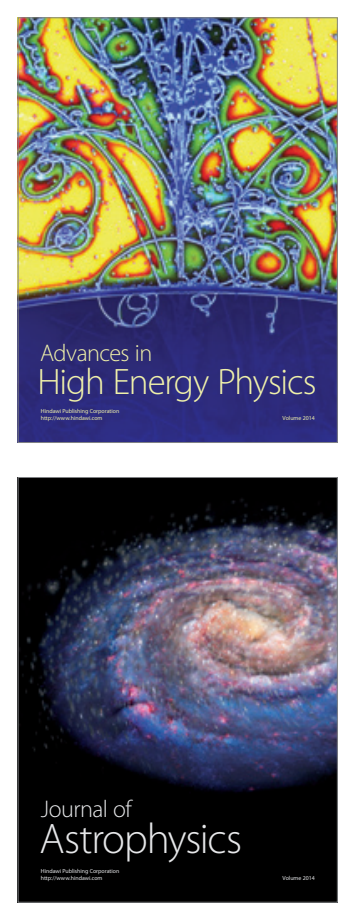
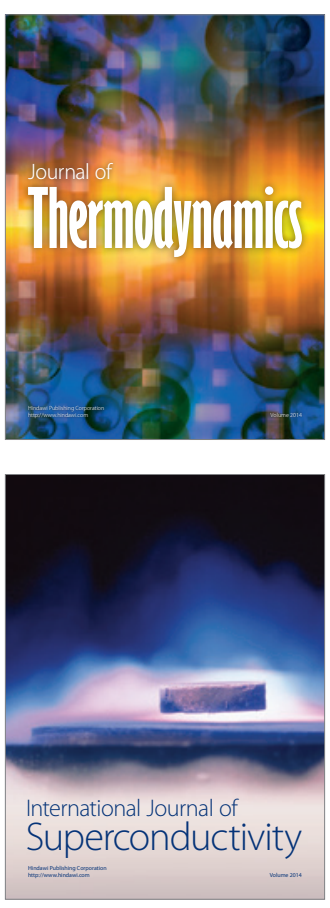
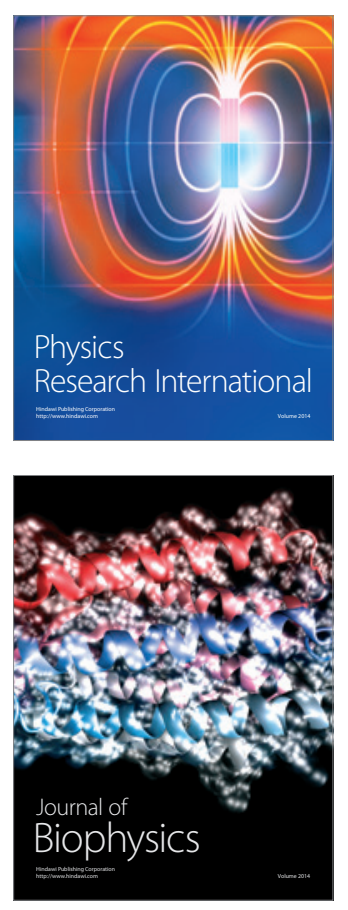
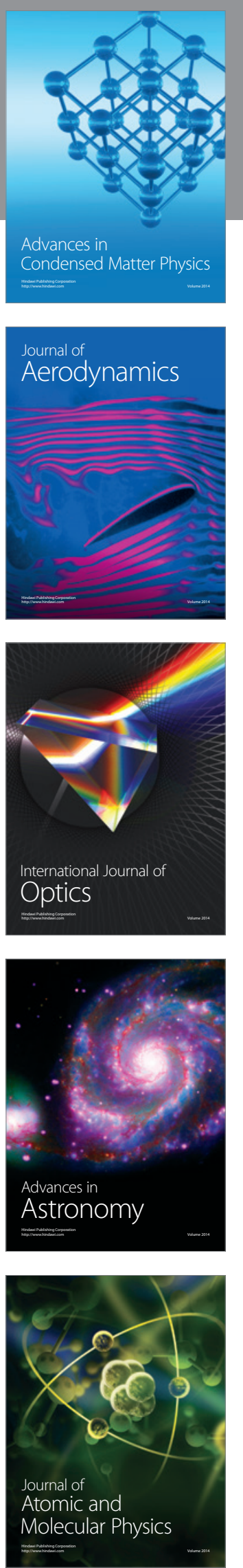